\title{
Genetic characterization and authentication of Dimocarpus longan Lour. using an improved RAPD technique
}

\author{
Z.Q. Mei ${ }^{1,2 *}$, S.Y. Fu ${ }^{3 *}$, H.Q. Yu ${ }^{1,2 *}$, L.Q. Yang ${ }^{1}$, C.G. Duan ${ }^{1}$, X.Y. Liu ${ }^{1}$, \\ S. Gong ${ }^{1}$ and J.J. Fu ${ }^{1}$ \\ ${ }^{1}$ Research Center for Preclinical Medicine, Luzhou Medical College, \\ Luzhou, Sichuan Province, China \\ ${ }^{2}$ Medicinal Botanical Association of Zhongshan Mountain, \\ Luzhou Medical College, Luzhou, Sichuan Province, China \\ ${ }^{3}$ Michael E. DeBakey High School for Health Professions, \\ Houston, TX, USA \\ *These authors contributed equally to this study. \\ Corresponding author: J.J. Fu \\ E-mail: fujunjiang@hotmail.com
}

Genet. Mol. Res. 13 (1): 1447-1455 (2014)

Received January 7, 2013

Accepted September 27, 2013

Published March 6, 2014

DOI http://dx.doi.org/10.4238/2014.March.6.3

\begin{abstract}
Dimocarpus longan Lour. is an edible and traditional herb in China, commonly referred to as longon. An improved randomly amplified polymorphic DNA (RAPD) protocol was here developed in order to determine the geographical origins of D. longan samples collected from 5 provinces in the southern and southwestern areas of China, including Sichuan, Hainan, Fujian, Guangdong, and Guangxi. Generally, the improved RAPD method generated good fingerprinting of the 5 samples using the selected 17 primers. In particular, primers SBS-A5, SBS-A13, SBS-I9, SBS-I20, SBS-M1, and SBS-Q12 produced distinguishable bands that clearly separated all 5 cultivars, suggesting that there are variations in RAPD genetic sites among the samples. The similarity index ranged from 0.69 to 0.76 . The Sichuan and Hainan clades clustered together with a 0.73 similarity index. The
\end{abstract}


Guangxi and Fujian clades clustered together with a 0.76 similarity index, and they formed the sister clade to the Sichuan/Hainan clade with a 0.71 similarity index. The Guangdong clade was in a basal polytomy with a 0.70 similarity index. Based on the abundant DNA polymorphisms, these longan accessions are distinguishable using our improved RAPD technique. Therefore, RAPD analysis is an effective technique in distinguishing the geographical origins of $D$. longan. Moreover, the improved method could also be employed for a variety of applications including genetic diversity and fingerprinting analyses.

Key words: Dimocarpus longan Lour.; RAPD; Authentication; Geographical origin

\section{INTRODUCTION}

Dimocarpus longan Lour., commonly known as longan, is a commercially attractive fruit that is widely distributed in subtropical areas. China has an over 2000 year old history of longan cultivation. In China, D. longan is grown in the provinces of Guangdong, Guangxi, Fujian, Sichuan, Yunnan, and Hainan. Longan production is dominant in Guandong, Guangxi, and Fujian, although D. longan is also grown in several areas of Luzhou city in Sichuan. Despite its own high production of the crop, China is a net importer of $D$. longan. As a traditional herb, longan fruit is used for enhancing memory (Park et al., 2010), promoting blood metabolism, relieving insomnia, and preventing amnesia (Yi et al., 2011). In addition, its secondary metabolic products have been shown to have antioxidative (Okuyama et al., 1999; Rangkadilok et al., 2005, 2007; Sun et al., 2007; Jiang et al, 2009), antiobesity (Yang et al., 2010), anticancer (Chung et al., 2010), antityrosinase (Prasad et al., 2010), and immunomodulatory activities (Su et al., 2010; Zhong et al., 2010; Yi et al., 2011).

Several molecular markers have been developed and applied since 1980, including random amplified polymorphic DNA (RAPD) (Williams et al., 1990; Devaiah and Venkatasubramanian, 2008; Ruzicka et al., 2009; Yazbeck et al., 2011; Noormohammadi et al., 2013), inter-simple sequence repeats (Feofilov et al., 2011; Ganopoulos et al., 2011; Noormohammadi et al., 2013), and amplified fragment length polymorphism (Vos et al., 1995). These molecular markers have been extensively utilized across various fields for assessments of genetic diversity, genotype fingerprinting, and molecular-assisted breeding.

D. longan from the city of Luzhou, in Sichuan Province along the Yangtze River, where it is also called Deng Gui, is commonly considered to be of greater value and is marketed at higher prices due to its fine, delicate scent and sweeter taste than those grown in Guangdong, Guangxi, and Fujian. Thus, it is essential to authenticate Luzhou longan cultivars from others. In this study, RAPD analysis was carried out to identify the geographical origin of longan samples from 5 different provinces. Usually, RAPD analyses are restricted due to the low stability and reproducibility of the technique. Fu et al. (2000) demonstrated that the resolution and production of RAPD markers might be greatly enhanced by prolonging the ramp time between the annealing and extension stages. The aims of this study were to develop an improved RAPD method for longan, and to examine the effectiveness of this improved method for discriminating Luzhou longan from other market varieties. We further discuss specialized local genotypes and evaluate the RAPD site variations among them. 


\section{MATERIAL AND METHODS}

\section{Plant materials}

The sources of the 5 longan accessions used in the RAPD analysis are listed in Table 1: Hainan, Sichuan, Guangxi, Guangdong, and Fujian Provinces (HN, SC, GX, GD, and FJ, respectively). All accessions were collected from typical places in Luzhou city in Sichuan, Dongwan city in Guangdong, Yulin city in Guangxi, Wanning city in Hainan, and Quanzhou city in Fujian (Figure 1). The plants were carefully identified and the specimens have been deposited at the source bank of the Luzhou Medical College.

\begin{tabular}{|c|c|c|c|}
\hline Sample & Common name & Sources & Accession No \\
\hline $\mathrm{HN}$ & Longan & Wanning/Hainan & GY001 \\
\hline $\mathrm{SC}$ & Longan & Luzhou/Sichuan & GY002 \\
\hline GX & Longan & Yulin/Guangxi & GY003 \\
\hline GD & Longan & Dongwan/Guangdong & GY004 \\
\hline FJ & Longan & Quanzhou/Fujian & GY005 \\
\hline
\end{tabular}

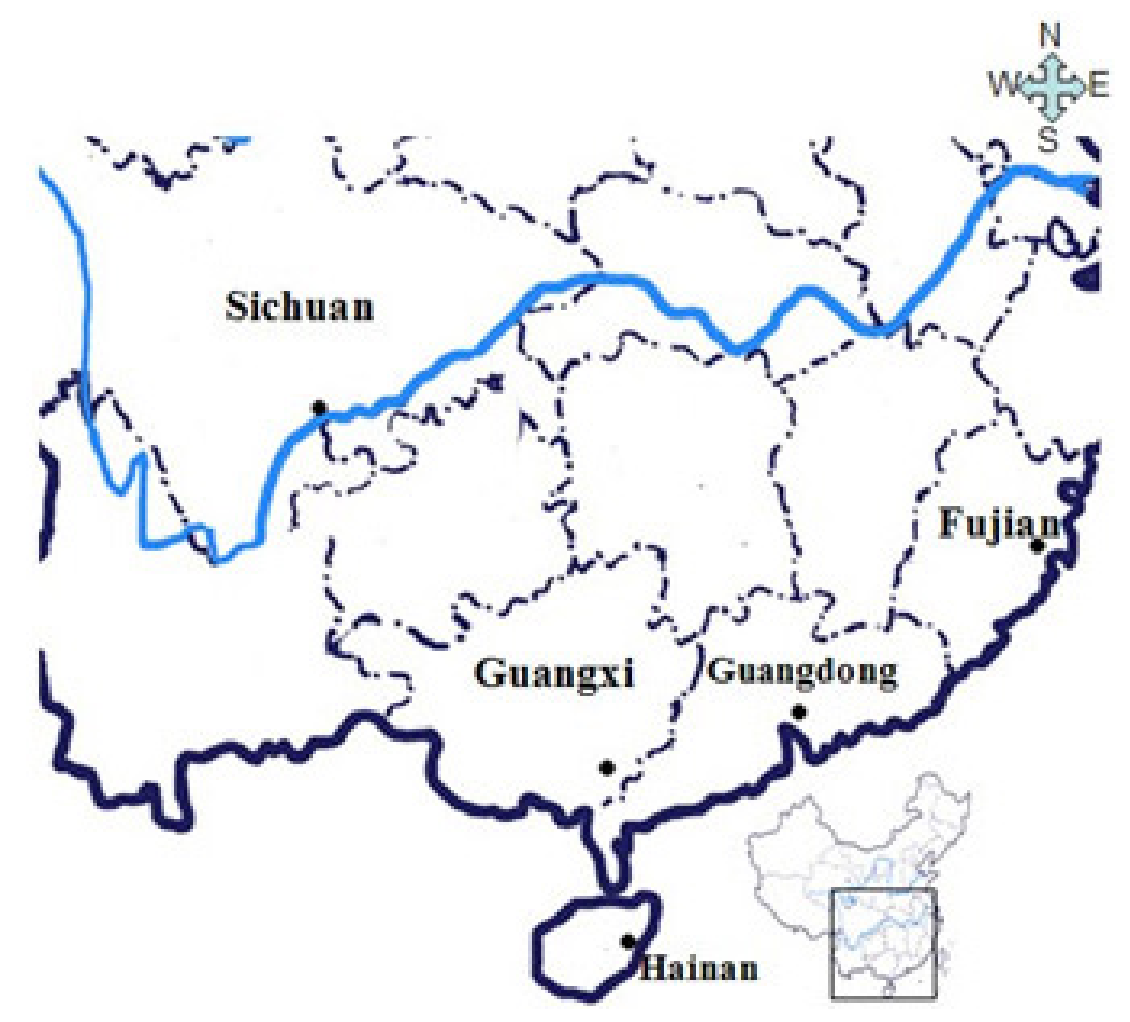

Figure 1. Localities of Dimocarpus longan samples from Luzhou (Sichuan), Dongwan (Guangdong), Yulin (Guangxi), Quanzhou (Fujian), and Wanning (Hainan), respectively, in China. Directions are indicated in the upper right. The spots in blue indicate the cities and the line in green indicate the Yangzi River. 


\section{DNA extraction and purification}

Fresh leaf samples were collected from plants of each accession and DNA was extracted using a newly developed method (Sharma et al., 2010). DNA quality was determined after electrophoresis on $1 \%$ agarose gels $(\mathrm{Fu}, 2012)$. DNA concentration was measured by spectrophotometry at 260 and $280 \mathrm{~nm}(\mathrm{Fu}, 2012)$. The final concentration of all DNA samples for PCR analysis was adjusted to $10 \mathrm{ng} / \mu \mathrm{L}$.

\section{RAPD PCR amplification and electrophoresis}

The primers used in the RAPD analysis are listed in Table 2; they were synthesized at Beijing SBS Genetech Co. Ltd., China. PCR was carried out using the SBS primer sets $\mathrm{A}, \mathrm{I}, \mathrm{M}, \mathrm{N}$, and Q. RAPD was performed in a total volume of $20 \mu \mathrm{L}$ containing $30 \mathrm{ng}$ DNA, $1 \mathrm{X}$ reaction buffer, $2 \mathrm{mM} \mathrm{MgCl}, 0.25 \mu \mathrm{M}$ of each primer, $200 \mu \mathrm{M}$ of each dNTP (TaKaRa Biotechnology, Dalian Co. Ltd., China), 1 U rTaq DNA polymerase (TaKaRa), and sterile water to the final volume. PCR amplification was performed as follows: initial denaturation at $95^{\circ} \mathrm{C}$ for $90 \mathrm{~s}$; followed by 40 cycles of $40 \mathrm{~s}$ at $94^{\circ} \mathrm{C}, 90 \mathrm{~s}$ at $36^{\circ} \mathrm{C}, 90 \mathrm{~s}$ at $72^{\circ} \mathrm{C}$; and a final extension of $5 \mathrm{~min}$ at $72^{\circ} \mathrm{C}$. PCRs of each accession were performed in a Mastercycler 5331 system (Eppendorf, Germany). The amplified PCR products were resolved by electrophoresis on $1.8 \%$ agarose gel in $1 \mathrm{X}$ TAE buffer. Gels were visualized with $0.5 \mu \mathrm{g} / \mathrm{mL}$ ethidium bromide staining and the images were documented using ChemiDoc XRS (Bio-Rad, USA) (Fu, 2012). Bands that were unambiguous and reproducible in successive amplifications were selected for scoring. An improved method for increasing the efficiency of the RAPD technique was also applied to compare the resolution and production of the 2 methods. The new technique involved adjusting the ramp time from annealing to extension from 3 to $0.3^{\circ} \mathrm{C} / \mathrm{s}$ (Fu et al., 2000). All PCRs were repeated at least 5 times for each of the 5 samples.

\begin{tabular}{|c|c|c|c|}
\hline Primer & Sequence & Primer & Sequence \\
\hline SBS-A7 & GAAACGGGTG & SBS-A9 & GGGTAACGCC \\
\hline SBS-A14 & TCTGTGCTGG & SBS-A15 & TTCCGAACCC \\
\hline SBS-A16 & AGCCGGCGAA & SBS-I9 & TGGAGAGCAG \\
\hline SBS-I1 & ACCTGGACAC & SBS-I20 & AAAGTGCGGG \\
\hline SBS-I2 & GGAGGAGAGG & SBS-N5 & ACTGAACGCC \\
\hline SBS-M1 & GTTGGTGGCT & SBS-N15 & CAGCGACTGT \\
\hline SBS-Q1 & GGGACGATGG & SBS-Q9 & GGCTAACCGA \\
\hline SBS-Q12 & AGTAGGGCAC & SBS-Q18 & AGGCTGGGTG \\
\hline SBS-Q19 & CССССТАТCA & & \\
\hline
\end{tabular}

\section{Band scoring and data analysis}

Bands in the gel profiles were recorded as present (1) or absent (0). The similarity matrix and the similarity index were calculated using the simple matching coefficient. The dendrogram was based on the unweighted pair group method with arithmetic mean, and the algorithm (UPGMA) was generated using the SAHN module in NTSYS pc 2.1 package. 


\section{RESULTS}

All accessions were collected from typical places in Luzhou city in Sichuan, Dongwan city in Guangdong, Yulin city in Guangxi, Wanning city in Hainan, and Quanzhou city in Fujian (Figure 1). RAPD analysis was carried out to identify the geographical origins of the longan samples from the 5 provinces. Three primers demonstrated similar repeatable fingerprints, but their low stability and reproducibility restricted the use of the regular RAPD technique (Figure 2A, B, and C; left panels). When the ramp time from annealing to extension was adjusted from $3^{\circ}$ to $0.3^{\circ} \mathrm{C} / \mathrm{s}$, resolution and production were markedly increased in the 5 longan samples (Figure 2; right panels).
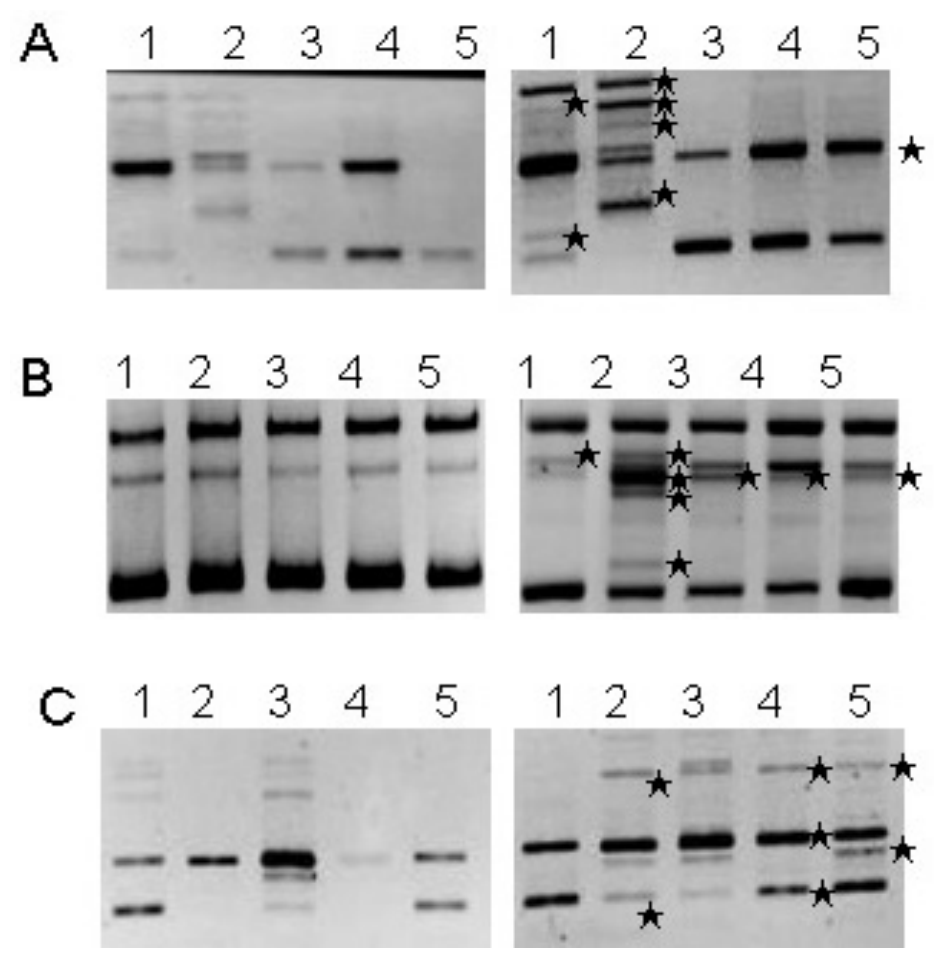

Figure 2. Comparison for RAPD profiles of five Dimocarpus longan samples generated by the primers SBS-A15, SBS-Q9, SBS-Q19 (A-C) with a $3^{\circ} \mathrm{C} / \mathrm{s} \mathrm{ramp} \mathrm{(left)} \mathrm{and} \mathrm{a} 0.3^{\circ} \mathrm{C} / \mathrm{s}$ (right). The "stars" in the right panels show the extra bands generated, indicating the increasing of the resolution and production with the new method. Lanes 1-5 = samples from Hainan, Sichuan, Guangxi, Guangdong, and Fujian, respectively.

Specifically, in the SBS-A15 amplification patterns, the band numbers and products were substantially enhanced in Hainan longan (Figure 2A; lane 1). In Sichuan Province, products increased from 2 to 6 (Figure 2A; lane 2), and in the Fujian sample the increase was from 1 to 2 (Figure 2A; lane 5). Primer SBS-Q9 showed the same 3 bands in all longan samples using the $3^{\circ} \mathrm{C} / \mathrm{s}$ ramp time (Figure $2 \mathrm{~B}$; left panel). However, fragment numbers increased when using a $0.3^{\circ} \mathrm{C} / \mathrm{s}$ ramp time; 6 fragments were obtained in the Sichuan samples, and 4 fragments were obtained in the other longan samples (Figure 2B; left panel). Similarly, 
band numbers and products increased using a $0.3^{\circ} \mathrm{C} / \mathrm{s}$ ramp time with the primer SBS-Q19 (Figure $2 \mathrm{C}$ ). Furthermore, no bands were generated in Guangdong longan under a $3^{\circ} \mathrm{C} / \mathrm{s}$ ramp time (Figure 2C; left panel), whereas this sample produced 3 bands when the ramp time was adjusted to $0.3^{\circ} \mathrm{C} / \mathrm{s}$ (Figure 2C; right panel). Therefore, the improved RAPD technique enabled the production of more reproducible polymorphic amplification bands compared with the traditional RAPD analysis technique.

Based on these results, this improved RAPD technique was applied to the remaining subset of the typical samples from the 5 provinces using RAPD primers. A total of 100 SBS primers were selected for polymorphism, and 17 primers (Table 2) generated reproducible polymorphic amplification bands. This indicated that these 17 primers were useful for identifying DNA polymorphisms and determining genetic relationships among the longan samples.

The representative characteristic bands and representative fingerprints of the 5 samples produced by these 17 primers are shown in Figures 3 to 5. Amplification patterns with primers SBS-N15 and SBS-Q18 differed among the 5 longan samples (Figure 3). The amplification patterns of Sichuan longan were similar to those of Guangdong (Figure 4A, C, and D) and Guangxi (Figure 4B) when the primers SBS-I1, SBS-A14, and SBS-Q1 were used (Figure 4), but differed from the other samples. Primers SBS-A5, SBS-A13, SBS-I9, SBS-I20, SBSM1, and SBS-Q12 produced distinguishable patterns and specific bands that clearly identified Luzhou longan from the others (identified by the arrows in Figure 5).
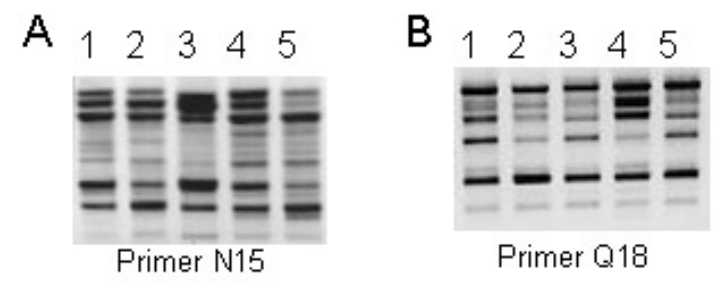

Primer Q18

Figure 3. Similar RAPD banding patterns obtained with the primers SBS-N15 and SBS-Q18 (A and B). Lanes 1-5 = samples from Hainan, Sichuan, Guangxi, Guangdong, and Fujian, respectively.
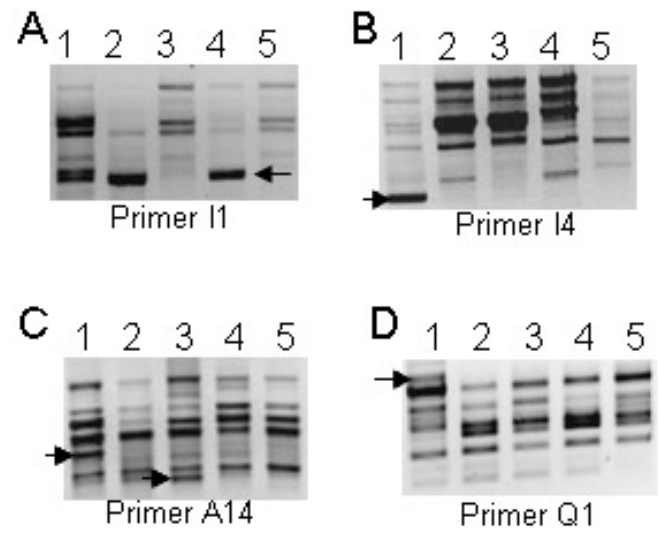

Figure 4. RAPD banding patterns obtained with the primers SBS-I1, SBS-I4, SBS-I20, SBS-A14 and SBS-Q1 (AD). The patterns in the Luzhou Dimocarpus longan sample were different from the other three places. Lanes 1-5 = samples from Hainan, Sichuan, Guangxi, Guangdong and Fujian, respectively. Arrows indicate different RAPD patterns and specific band fragments. 

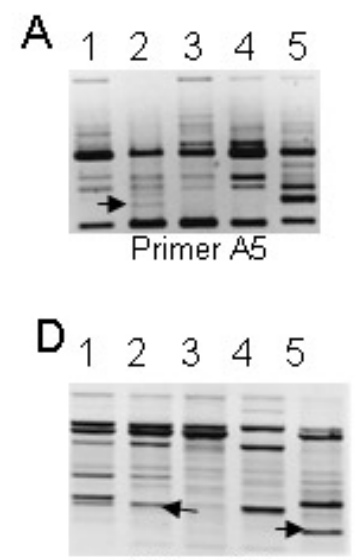

Primer 120

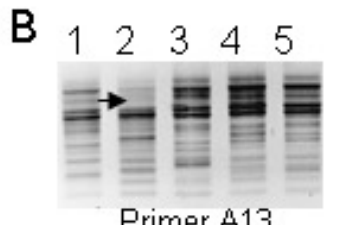

Primer A13

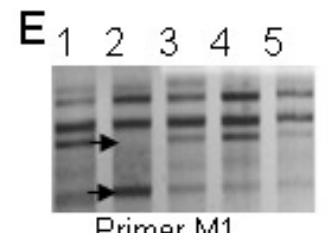

Primer M1

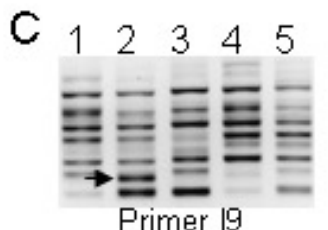

Primer 19

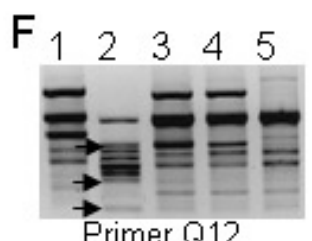

Figure 5. Different RAPD banding patterns in the Luzhou Dimocarpus longan sample obtained with the primers SBS-A5, SBS-A13, SBS-I9, SBS-I20, SBS-M1, and SBS-Q12 (A-F). Lanes 1-5 = samples from Hainan, Sichuan, Guangxi, Guangdong, and Fujian, respectively. Arrows indicate different RAPD patterns with specific band fragments in the Luzhou $D$. longan sample.

The number of amplified fragments ranged from 2 (SBS-Q19) to 15 (SBS-A13) across the accessions tested, and band sizes ranged from approximately 250 to $2000 \mathrm{bp}$. The 17 primers produced a total of 87 bands with an average of 5.11 per primer. Thirty-two percent of the 87 generated bands were polymorphic. These results illustrated that DNA polymorphisms could be detected among the 5 longan cultivars.

Based on scoring of the 87 bands, the similarity matrix was used to determine genetic relationships. The resulting dendrogram, based on a UPGMA algorithm, is displayed in Figure 6. The similarity coefficients ranged from 0.69 to 0.76 (Table 3). Sichuan and Hainan clades clustered together with a 0.73 similarity index, while Guangxi and Fujian clades clustered together with a 0.76 similarity index, and formed a sister to the Sichuan/Hainan clade with a 0.71 similarity index. The Guangdong clade was in a basal polytomy with a 0.70 similarity index.

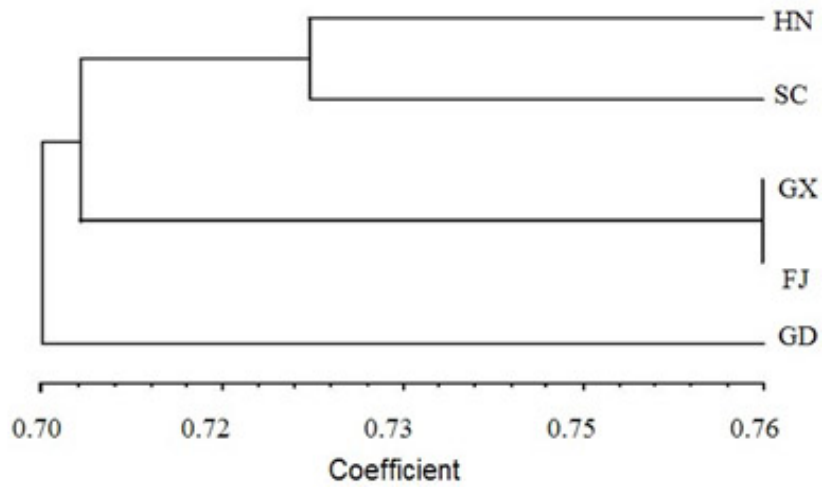

Figure 6. Dendrogram of the five Dimocarpus longan samples based on 87 PCR bands amplified by the 17 primers. HN, SC, GX, GD, and FJ represent samples from Wanning (Hainan), Luzhou (Sichuan), Yulin (Guangxi), Dongwan (Guangdong), and Quanzhou (Fujian), respectively. Bar on the bottom indicates similarity index based on the similarity matrix coefficient. 


Table 3. Similarity index of RAPD fingerprints generated by the 17 primers.
\begin{tabular}{llllll}
\hline Sample & HN & SC & GX & GD & FJ \\
\hline HN & 1 & - & - & - & - \\
SC & 0.725 & 1 & 1 & - & - \\
GX & 0.725 & 0.7 & 0.7125 & 1 & - \\
GD & 0.7125 & 0.6875 & 0.7625 & 0.7 & 1 \\
FJ & 0.7125 & 0.6875 & &
\end{tabular}

For abbreviations, see Table 1.

\section{DISCUSSION}

RAPD and cluster analysis is a useful tool for providing valuable data in determining relationships among different populations (Crawford, 1990; Williams et al., 1990). Based on scoring of the 87 bands by RAPD, the similarity matrix was used to determine genetic relationships. The similarity coefficients ranged from 0.69 to 0.76 . Sichuan and Hainan clades clustered together with a 0.73 similarity index, while Guangxi and Fujian clades clustered together with a 0.76 similarity index, and formed a sister to the Sichuan/Hainan clade with a 0.71 similarity index. The Guangdong clade was in a basal polytomy with a 0.70 similarity index. Therefore, based on the abundant DNA polymorphisms identified using our improved RAPD technique, the longan accessions are clearly distinguishable.

In the dendrogram of the 5 cultivars, Guangxi longan was found to be more closely related to the Fujian samples than to the others. Although there is geographic isolation between Sichuan and Hainan longan, they were clustered together. This suggested that the genetic relationship among the 5 accessions is not always correlated to their geographic localities. These results suggest that the Sichuan longan might have originated from Hainan. Furthermore, longan from the Luzhou city of Sichuan Province along the Yangtze River is commonly considered to be of better quality and achieves higher prices on the market than the other cultivars, suggesting that this improved quality might depend on characteristics of the soil and climate in this region.

The template quality, cycle numbers, and primer concentrations can all affect the resolution and repetition of RAPD analyses. Fu et al. (2000) reported that RAPD resolution and production are greatly increased by prolonging the ramp time from annealing to extension in spider DNA amplification. In the present study, this improved RAPD method, adjusting the ramp parameter to $0.3^{\circ} \mathrm{C} / \mathrm{s}$, was effective in revealing genetic characterizations of longan by generating more and/or stronger bands with clear, reproducible, and distinguishable patterns (Figure 2).

In RAPD experiments, the reliability and stability of fingerprints mostly depends on the complexity of genomic DNA (Carl et al., 1995). The amplification repetitions guaranteed RAPD reproducibility in the current study. Different longan genotypes were revealed by specific or polymorphic bands. Therefore, the improved RAPD technique of prolonging the ramp time to increase resolution and production is useful for the identification of different longan cultivars, and could be applied in a variety of fields, particularly for genetic diversity and fingerprinting analyses in plants.

\section{ACKNOWLEDGMENTS}

Research supported in part by the National Natural Science Foundation of China (\#30371493 and \#81172049), the Research Foundation of Luzhou City (\#2011-S-30), and a 
Starting Package from Luzhou Medical College. The authors particularly thank all individuals who provided longan leaves.

\section{REFERENCES}

Carl WD, Dieffenbach and Gabriela SD (1995). PCR Primer: A Laboratory Manual. Cold Spring Harbor Laboratory Press, New York.

Chung YC, Lin CC, Chou CC and Hsu CP (2010). The effect of Longan seed polyphenols on colorectal carcinoma cells. Eur. J. Clin. Invest. 40: 713-721.

Crawford DJ (1990). Plant Molecular Systematics: Macromolecular Approaches. Wiley, New York.

Devaiah KM and Venkatasubramanian P (2008). Genetic characterization and authentication of Embelia ribes using RAPD-PCR and SCAR marker. Planta Med. 74: 194-196.

Feofilov AV, Bardukov NV and Glazko VI (2011). Gene pool differentiation between Altaic and trotting horse breeds inferred from ISSR-PCR marker data. Genetika 47: 1230-1235.

Fu JJ (2012). Short Protocols in Medical Molecular Biology. China Medical Science Press, Beijing.

Fu JJ, Li LY, Xu X, Wang Z, et al. (2000). An improved method for increasing the efficiency of the technique of random amplified polymorphic DNA (RAPD). Hereditas 22: 251-252.

Ganopoulos I, Merkouropoulos G, Pantazis S, Tsipouridis C, et al. (2011). Assessing molecular and morpho-agronomical diversity and identification of ISSR markers associated with fruit traits in quince (Cydonia oblonga). Genet. Mol. Res. 10: 2729-2746.

Jiang G, Jiang Y, Yang B, Yu C, et al. (2009). Structural characteristics and antioxidant activities of oligosaccharides from longan fruit pericarp. J. Agric. Food Chem. 57: 9293-9298.

Noormohammadi Z, Hasheminejad-Ahangarani FY, Sheidai M, Ghasemzadeh-Baraki S, et al. (2013). Genetic diversity analysis in Opal cotton hybrids based on SSR, ISSR, and RAPD markers. Genet. Mol. Res. 12: 256-269.

Okuyama E, Ebihara H, Takeuchi H and Yamazaki M (1999). Adenosine, the anxiolytic-like principle of the Arillus of Euphoria longana. Planta Med. 65: 115-119.

Park SJ, Park DH, Kim DH, Lee S, et al. (2010). The memory-enhancing effects of Euphoria longan fruit extract in mice. J. Ethnopharmacol. 128: 160-165.

Prasad KN, Yang B, Shi J, Yu C, et al. (2010). Enhanced antioxidant and antityrosinase activities of longan fruit pericarp by ultra-high-pressure-assisted extraction. J. Pharm. Biomed. Anal. 51: 471-477.

Rangkadilok N, Worasuttayangkurn L, Bennett RN and Satayavivad J (2005). Identification and quantification of polyphenolic compounds in Longan (Euphoria longana Lam.) fruit. J. Agric. Food Chem. 53: 1387-1392.

Rangkadilok N, Sitthimonchai S, Worasuttayangkurn L, Mahidol C, et al. (2007). Evaluation of free radical scavenging and antityrosinase activities of standardized longan fruit extract. Food Chem. Toxicol. 45: 328-336.

Ruzicka J, Lukas B, Merza L, Gohler I, et al. (2009). Identification of Verbena officinalis based on ITS sequence analysis and RAPD-derived molecular markers. Planta Med. 75: 1271-1276.

Sharma P, Joshi N and Sharma A (2010). Isolation of genomic DNA from medicinal plants without liquid nitrogen. Indian J. Exp. Biol. 48: 610-614.

Su DX, Zhang MW, Liao ST and Hou FL (2010). Effects of water soluble extracts from longan on immune regulation in normal mice. China Agr. Sci. 43: 1919-1925.

Sun J, Shi J, Jiang Y, Xue SJ, et al. (2007). Identification of two polyphenolic compounds with antioxidant activities in longan pericarp tissues. J. Agric. Food Chem. 55: 5864-5868.

Vos P, Hogers R, Bleeker M, Reijans M, et al. (1995). AFLP: a new technique for DNA fingerprinting. Nucleic Acids Res. 23: 4407-4414.

Williams JG, Kubelik AR, Livak KJ, Rafalski JA, et al. (1990). DNA polymorphisms amplified by arbitrary primers are useful as genetic markers. Nucleic Acids Res. 18: 6531-6535.

Yang DJ, Chang YY, Hsu CL, Liu CW, et al. (2010). Antiobesity and hypolipidemic effects of polyphenol-rich longan (Dimocarpus longans Lour.) flower water extract in hypercaloric-dietary rats. J. Agric. Food Chem. 58: 2020-2027.

Yazbeck GM, Brandao RL, Cunha HM and Paglia AP (2011). Detection of two morphologically cryptic species from the cursor complex (Akodon spp; Rodentia, Cricetidae) through the use of RAPD markers. Genet. Mol. Res. 10: 2881-2892.

Yi Y, Liao ST, Zhang MW, Shi J, et al. (2011). Physicochemical characteristics and immunomodulatory activities of three polysaccharide-protein complexes of longan pulp. Molecules 16: 6148-6164.

Zhong K, Wang Q, He Y and He X (2010). Evaluation of radicals scavenging, immunity-modulatory and antitumor activities of longan polysaccharides with ultrasonic extraction on in S180 tumor mice models. Int. J. Biol. Macromol. 47: $356-360$. 